\title{
Reply to "Mechanisms of postoperative progression of steno-occlusive lesions in the posterior cerebral artery in moyamoya disease"
}

\author{
Ji Yeoun Lee $\cdot$ Kyu-Chang Wang
}

Received: 4 February 2014 / Accepted: 5 February 2014 / Published online: 20 February 2014

(C) Springer-Verlag Berlin Heidelberg 2014

We thank Dr. Mugikura and his colleague for their comments on our paper "Posterior cerebral artery involvement in moyamoya disease: initial infarction and angle between PCA and basilar artery." They have listed in detail three possible mechanisms that may bring about the progression of posterior cerebral artery (PCA) lesions in moyamoya disease (MMD): "primary progression" steno-occlusion of PCA, involution of PCA due to development of sufficient collaterals from the revascularization of anterior circulation, or occlusion of PCA due to presence of major infarctions. The latter two commonly hypothesize that the steno-occlusion of PCA is a secondary phenomenon of decrease in vascular demand from the PCA due to the reasons mentioned.

We totally agree with their idea that the delayed progression of PCA is a primary phenomenon, related to the nature of the disease. As we mentioned in our paper, although the presence of preoperative infarction was strongly associated with progression of the PCA stenosis, this should not be interpreted as a causal relationship but as the concurrence of the two most influential factors affecting the prognosis of MMD. As our clinical experience with follow-up of MMD patients accumulate, we feel that many patients with PCA progression are "symptomatic" which means that the progression is not just the result of decreased demand of collaterals from the PCA but a real progression of ischemia of the PCA area. We agree that the second and the third mechanisms may accelerate the stenosis of PCAs. However, our indication for additional revascularization surgery of the PCA area requires presence and dominance of the first mechanism proposed in this commentary.

Our paper focused on the clinical aspects, especially the preoperative factors related to delayed progression of PCA stenosis in MMD. The commentary by Dr. Mugikura and his colleague has greatly enriched the academic discussion on the topic of posterior circulation involvement in MMD.
J. Y. Lee $\cdot$ K.-C. Wang $(\bowtie)$

Division of Pediatric Neurosurgery, Seoul National University

Children's Hospital, Seoul, South Korea

e-mail: kcwang@snu.ac.kr 